\title{
A LES-Langevin model for turbulence
}

\author{
J.-P. Laval ${ }^{1}$, B. Dubrulle ${ }^{2}$ \\ ${ }^{1}$ Laboratoire de Mécanique de Lille, CNRS, UMR 8107,Blv Paul Langevin, \\ F-59655 Villeneuve d'Ascq Cedex, France \\ ${ }^{2}$ Groupe Instabilités et Turbulence, \\ CNRS, URA 2464, SPEC/DRECAM/DSM/CEA Saclay, \\ F-91191 Gif sur Yvette, Cedex
}

October 16, 2018

\begin{abstract}
We propose a new model of turbulence for use in large-eddy simulations (LES). The turbulent force, represented here by the turbulent Lamb vector, is divided in two contributions. The contribution including only subfilter fields is deterministically modeled through a classical eddy-viscosity. The other contribution including both filtered and subfilter scales is dynamically computed as solution of a generalized (stochastic) Langevin equation. This equation is derived using Rapid Distortion Theory (RDT) applied to the subfilter scales. The general friction operator therefore includes both advection and stretching by the resolved scale. The stochastic noise is derived as the sum of a contribution from the energy cascade and a contribution from the pressure. The LES model is thus made of an equation for the resolved scale, including the turbulent force, and a generalized Langevin equation integrated on a twice-finer grid. We compare the full model with several approximations. In the first one, the friction operator of the Langevin equation is simply replaced by an empirical constant, of the order of the resolved scale correlation time. In the second approximation, the integration is replaced by a condition of instantaneous adjustment to the stochastic force. In this approximation, our model becomes equivalent to the velocity-estimation model of Domaradzki et al. 7, 5, 8. In the isotropic, homogeneous situations we study, both approximations provide satisfactory results, at a reduced computational cost. The model is finally validated by comparison to DNS and is tested against classical LES models for isotropic homogeneous turbulence, based on eddy viscosity. We show that even in this situation, where no walls are present, our inclusion of backscatter through the Langevin equation results in a better description of the flow.
\end{abstract}

\section{Introduction}

The rationale for Large Eddy Simulation is often rooted in our inability to handle all the degrees of freedom of a large Reynolds number turbulent flow. Given that the smaller scales monopolize most of the computing resources, it is tempting to cut through the number of degrees of freedom via an ad hoc small-scale decimation. The price to pay is of course a need for parameterization (the so-called SubGrid Scale models, or SGS), to make up for the energy transfer of the ghost scales. We refer the reader to 3, 32, 28, 31] for recent reviews about modern parameterization strategies in LES. We may loosely divide the SGS models in two classes, according to their philosophy: the "deterministic" models based on eddyviscosities, and the "stochastic" models, based on synthetic fields. In eddy-viscosity methods, the action of small scales is parameterized via a few deterministic numbers, linked with the various components of the subgrid-scale stress tensor. These models seek to reproduce the intensification of energy transport due to the action of scales widely separated from the considered one. However, they fail to reproduce backward energy transfer (backscatter) from small to large scale, created by elongated triads in the spectral space 30]. This effect has been shown to induce a stochastic character in the LES [20. In ideal situations, where the turbulence is isotropic, homogeneous and far from wall, this backscatter is usually viewed as secondary, and eddy-viscosity based models are generally satisfactory. However, in more realistic situations, including turbulence near walls or in boundary layers, the energy backscatter has proven to 
reproduce a realistic backscatter in some situations $(15,23)$. The need for backscatter modeling also leads to the development of "stochastic" strategies, where the discarded small-scale motions are replaced by a set of random numbers, mimicking either a random force or synthetic velocity fields [7, 33, 17. In many ways, these strategies resemble the strategies used to describe the dynamics of a heavy particle coupled to a thermal bath involving many degrees of freedom (the so-called Brownian motion). The decimation is here performed by substituting in place of the bath a deterministic friction and a stochastic force, the two terms being linked through the dissipation theorem. The initial problem is then completely described through the so-called Langevin equation.

Turbulence is typically an out-of-equilibrium system, and there is probably no hope that such a simple description will ever be possible (would it be only because no fluctuation-dissipation theorem prevails in turbulence!). However, we would like to use this analogy to motivate a new strategy for LES modeling: replace the actual dynamics of the decimated degrees of freedom by a suitable noise, via a Langevin equation. Although this strategy may seem close to recent models based on synthetic fields, we would like to point out an important philosophical difference: rather than trying to estimate the actual small-scale dynamics, we aim at trying to estimate a plausible small-scale dynamics. We believe there is no unique solution for this last option. In the sequel, we present one solution based upon Rapid Distortion Theory [17. There may exist actually more efficient models, based e.g. upon information theory [16].

To be more specific, consider a turbulent flow, with velocity field $u_{i}(x, t)$ and introduce a filtering procedure so as to separate it into a resolved field $U_{i}=\overline{u_{i}}$ and a subfilter field $u_{i}^{\prime}=u_{i}-U_{i}$. The resolved field obeys a dynamical equation obtained by filtering of the Navier-Stokes equation, which may conveniently be written as 39 :

$$
\begin{aligned}
& \partial_{t} \mathbf{U}+\overline{(\mathbf{U} \cdot \nabla) \bar{U}}+\overline{\mathbf{l}}+\overline{\left(\mathbf{u}^{\prime} \cdot \nabla\right) \mathbf{u}^{\prime}}=-\nabla P+\nu \Delta \mathbf{U}, \\
& \mathbf{l}=(\mathbf{U} \cdot \nabla) \mathbf{u}^{\prime}+\left(\mathbf{u}^{\prime} \cdot \nabla\right) \mathbf{U} .
\end{aligned}
$$

Here, $P$ is the resolved pressure and $\nu$ is the viscosity and $\mathbf{l}$ is a turbulent force. In idealistic situations including a spectral gap between resolved and subfilter scale, the vector $\mathbf{l}$ is zero, and one can rigorously show that the contribution of the remaining term is of "diffusive" type (providing certain symmetries which exclude first order behavior such as the AKA effect [11]). Even as one departs from this idealistic situation, experimental [37] and numerical study [18] show that this term correlates strongly to the resolved velocity gradient, thereby allowing a deterministic treatment through an eddy-viscosity of appropriate shape. In the same time, the force vector 1 becomes increasingly non-negligible (it can even become dominant in 2D situations see [17]). It is responsible for backscatter type of behaviour and needs to be modeled through novel "non-diffusive" and "non-deterministic" strategies. In the sequel, we will therefore focus onto the modeling of the $\mathbf{l}$ term, via a generalized Langevin equation $\partial_{t} \mathbf{l}=A \mathbf{l}+\xi$ where $A$ is a generalized evolution operator, and $\xi$ is a noise.

However, a clear difficulty associated with this strategy is the lack of theoretical guide (equivalent of the statistical mechanics in Brownian motion) to help us devising the "best" friction, and the "best" stochastic force. For the time being, we then choose to pin down our model as best as possible to the real dynamics of Navier-Stokes by trying to derive it from the original dynamical equations, rather than from pure empirical or dimensional considerations. For this, we reformulate the RDT-Langevin model of Laval et al. 17] in a way suitable for LES. There are of course limitations to this approach, pertaining the need for both a simple enough model, and for tractable analytical computations. We try to formulate them as honestly as possible by pointing out the approximation we make at the various stage of the derivation of the model. This is done in Section [2 The LES-Langevin model is then implemented in the case of periodic three-dimensional turbulence and comparison with other existing LES models is provided in Section 3 Our conclusions follow in Section 4.

\section{The LES-Langevin model of turbulence}

\subsection{Derivation of the Langevin equation}

Our derivation is based on the stochastic RDT model developed by Laval et al. [19, 12, 17]. This model is based on the observation that subfilter-scales are mostly slaved to resolved scales via linear processes akin to rapid distortion. This property is substantiated by various numerical simulations, and is linked with the prominence of non-local interactions at subfilter-scale [17. Using incompressibility, the small-scale dynamics in this model can be written as:

$$
\partial_{t} \mathbf{u}^{\prime}=-\mathbf{l}^{\prime}-\nabla p^{\prime}+\nabla\left(\nu+\nu_{t}\right) \nabla \mathbf{u}^{\prime}-\mathbf{f} .
$$


subfilter scales and $\mathbf{f}$ is a forcing stemming from the energy cascade. The latter can be shown to be dominated by resolved scales non-linearities through $f_{i}=\partial_{j}\left(U_{i} U_{j}-\overline{U_{i} U_{j}}\right)$. Finally, we may use the observation that subfilter scales vary over fast time scale with respect to resolved scales to write:

$$
\begin{aligned}
\partial_{t} \mathbf{l} \approx & (\mathbf{U} \cdot \nabla) \partial_{t} \mathbf{u}^{\prime}+\left(\partial_{t} \mathbf{u}^{\prime} \cdot \nabla\right) \mathbf{U} \\
\approx & \left.-\left\{(\mathbf{U} \cdot \nabla)\left(\mathbf{l}^{\prime}+\mathbf{f}\right)\right)+\left[\left(\mathbf{l}^{\prime}+\mathbf{f}\right) \cdot \nabla\right] \mathbf{U}\right\} \\
& -\left\{(\mathbf{U} \cdot \nabla) \nabla p^{\prime}+\left[\left(\nabla p^{\prime}\right) \cdot \nabla\right] \mathbf{U}\right\} \\
& + \text { visc },
\end{aligned}
$$

where visc gathers all the term containing $\nu$ or $\nu_{t}$. This equation is only an approximation, in so far as the assumption of "rapidly varying scales" becomes less and less valid as the resolved scales and subfilter velocities are becoming closer and closer in scale space. However, it seems to capture the dominant physics of the evolution of the vector 1 , as will be later shown. Further, it is tempting to simplify the viscous terms of the second equation of (3) to try and get a closed equation for 1 . Indeed, these terms involve an a priori rather arbitrary turbulent viscosity and one could redefine it so that the viscous terms are simply lumped into a term $\nabla\left(\nu+\nu_{t}\right) \nabla \mathbf{l}$. Finally, we note that the terms involving the subfilter pressure depend on boundary conditions and on subfilter velocities, so that they can be thought to vary over a fast time scale, contrarily to the $\mathbf{f}$ term which varies over a slow time scale. We therefore choose to collect all the term involving the pressure and $\mathbf{f}$ into a noise term,$\xi_{\mathbf{0}}+\xi$, such that $\xi$ is a Gaussian-centered noise and

$$
\begin{aligned}
& \xi_{0}=-(\mathbf{U} \cdot \nabla) \mathbf{f}-(\mathbf{f} \cdot \nabla) \mathbf{U}, \\
& <\xi>=0 \\
& <\xi_{i}(t, x) \xi_{j}\left(t^{\prime}, x^{\prime}\right)>=T_{i j}\left(t-t^{\prime}, x-x^{\prime}\right),
\end{aligned}
$$

where $T_{i j}$ is the noise correlation function, to be specified. Note that since $\xi$ comes from a pressure contribution, it only affects the non-soleinodal part of the turbulent force. In the sequel, since we work with periodic boundary conditions, we can apply a simple projection procedure to work only with soleinodal fields, thereby discarding the $\xi$ term. We therefore leave the study of the influence of this term for future work, involving more realistic boundary conditions such as flow near walls.

Collecting all the results, we therefore obtain the following RDT based-model for the turbulent force 1 as:

$$
\partial_{t} \mathbf{l}=-(\mathbf{U} \cdot \nabla) \mathbf{l}^{\prime}-\left(\mathbf{l}^{\prime} \cdot \nabla\right) \mathbf{U}+\nabla\left(\nu+\nu_{t}\right) \nabla \mathbf{l}+\xi_{\mathbf{0}}+\xi
$$

It takes the form of a generalized Langevin equation, with friction made of viscosity and rapid distortion by resolved scales, and with stochastic forcing, with mean value generated through the energy cascade, and whose correlations are physically imposed by boundary conditions via pressure terms.

Before implementing this Langevin equation into a LES model, we first validate it through dynamical a priori tests and seek optimal performances by tuning of $\nu_{t}$. Our LES-Langevin model will follow after this validation step.

\subsection{Dynamical a priori testing}

\subsubsection{The numerical procedure}

The numerical simulations described in the present paper have been performed with a spectral code over a cubic domain. This choice is mainly motivated by its performances in terms of accuracy and its versatility regarding any variant of our model. The aliasing is removed by keeping only the $2 / 3$ largest modes in each direction. The time integration is performed with a second order Adams Bashforth scheme. The simulations with both the RDT and the LES-Langevin models are performed with a sharp cut-off filter at $k=k_{c}$. The filter may not be optimum in terms of efficiency, but it has the interesting properties to clearly separate scales and to commute with derivative operators. The influence of the filter would need to be conducted for a complete validation. The validation tests are performed on decaying and forced isotropic homogeneous turbulence. The simulation of decaying turbulence is initialized with a Gaussian velocity field with a given energy spectra $E\left(k, t_{o}\right) \propto k^{4} \exp \left(-k^{2} / 8\right)$. For the forced simulation, the forcing $\mathbf{F}$ is defined by $\mathbf{F}(\mathbf{k}, t) d t=\gamma(k, t) \mathbf{u}(\mathbf{k}, t)$, with $\gamma$ chosen so that the difference of the energy spectra before and after the forcing (i.e. the energy injection rate $\Im$ ) is constant in time $(\Im=1$.). The forcing is concentrated at the lowest wavenumbers $\mathbf{k}$ such as $0<k<1$. The initial time of the forced simulation is chosen so that statistical properties are stabilized. The simulations are integrated over approximately 6.3 
molecular viscosity of $\nu=0.0014$ is used for all simulations. The resulting Taylor Reynolds number is approximately constant and equal to $R_{\lambda}=200$ for the forced DNS and varies from $R_{\lambda}=260$ to $R_{\lambda}=26$ in the decaying case. Both DNS are performed with $341^{3}$ effective wavenumbers after desaliasing $\left(512^{3}\right.$ grid points). The space resolution allows a computation of scales down to 0.5 and 0.8 Kolmogorov scale for the decaying and the forced DNS respectively. The simulation with LES-Langevin model are performed with $k_{c}=21$ ( $42^{3}$ effective wavenumbers are used for the resolved scales).

\subsubsection{Validation of the model}

The validation of the model is conducted by dynamical tests performed with the DNS of decaying 3D turbulence. It is performed by comparing a full DNS and a simulation at the same resolution, in which the turbulent force $\mathbf{l}$ is replaced by the solution of (5). This way, we may explore the validity of the approximations we made in its derivation. We focus here on the energy spectra, so as to capture possible deficiency of our model regarding energy transfer between scales. The test is performed for $1<t<2$ (all the simulations are initialized with the same field at $t=1$ ). By that time, the kinetic energy is divided by a factor of approximately 2. The energy spectra at time $t=2$ are shown in fig. 1].

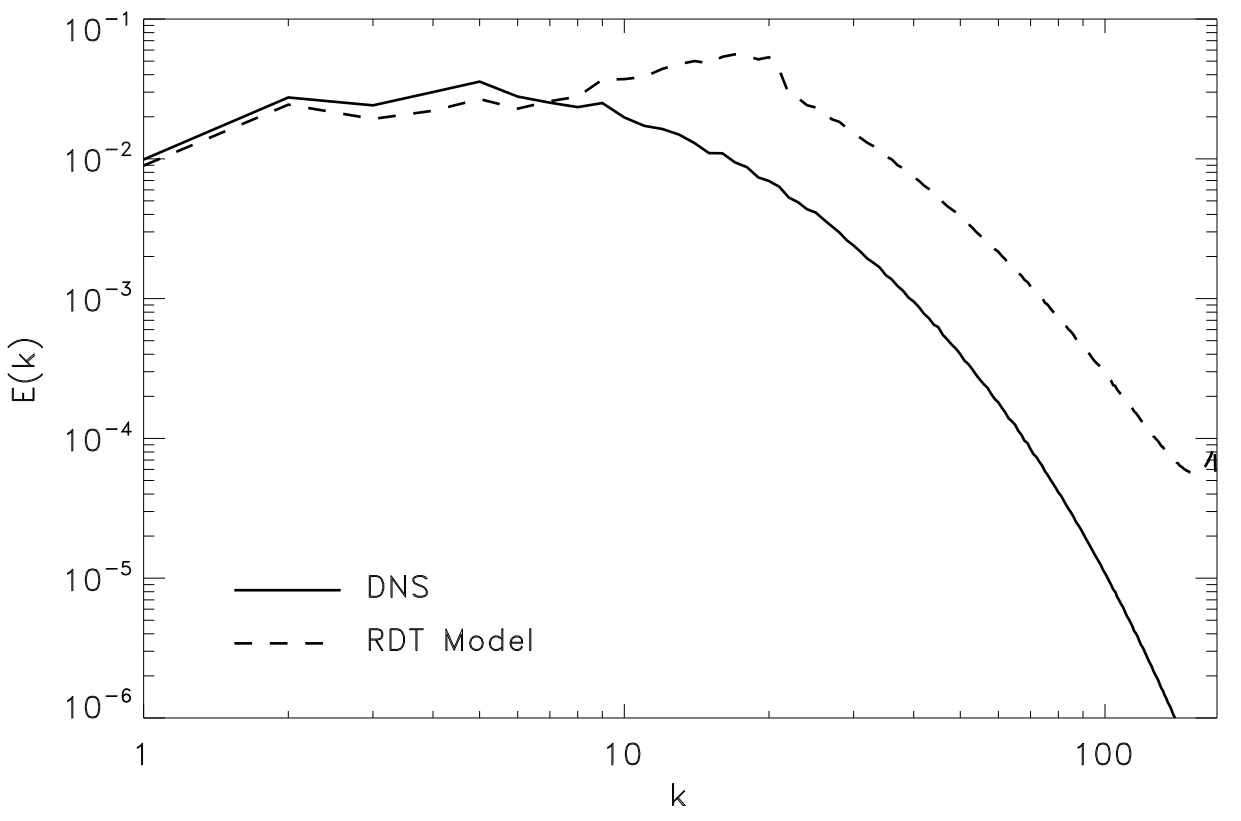

Figure 1: Energy spectra of the RDT Models (5) and the equivalent DNS at $t=2$ of simulations of decaying turbulence (the RDT simulation is initialized with the velocity field of the DNS at $t=1$ )

The model (RDT) and the DNS agree at the largest scales $(k<3)$ but they significantly differ for smaller scales (the bump at $\mathrm{k}=21$ is due to the coupling between the equation for resolved scales and the equation for subfilter scales running in parallel). This can be explained through the analysis of the evolution of 1 (fig. 21).

One sees that the RDT model leads to a constant increase of the smallest modes of $\mathbf{l}$ in time. After a given period of time, the contribution of these unrealistic resolved scales of $\mathbf{l}$ influence the model of the velocity field resolved scales.

An explanation of this feature can be found following a recent study of the RDT model (2) [12] showing that the process of small-scale stretching by random large scales is akin to a dynamo process, with exponential increase of the small-scale energy. A way to stabilize the system is to include a friction term in the RDT equation, leading to a stationary energy spectrum with index depending on the friction time $\tau_{f}$. A Kolmogorov $k^{-5 / 3}$ spectrum is obtained for $\tau_{f}=27 / 22 \Omega$, where $\Omega=\left\langle\left(S_{i j} S_{i j}\right)^{-1 / 2}\right\rangle$ is a typical stretching rate based on spatial average of the large-scale velocity stress tensor $S_{i j}$. Using eq. (3), one sees that such a friction term generates an equivalent friction term in the equation for the Lamb vector. This remark motivates the introduction of a stabilizing friction term $-\mathbf{l} / \tau_{f}$ in the equation for $\mathbf{l}$ to try and stabilize the coupled system. Indeed one observes a significant improvement with respect to 


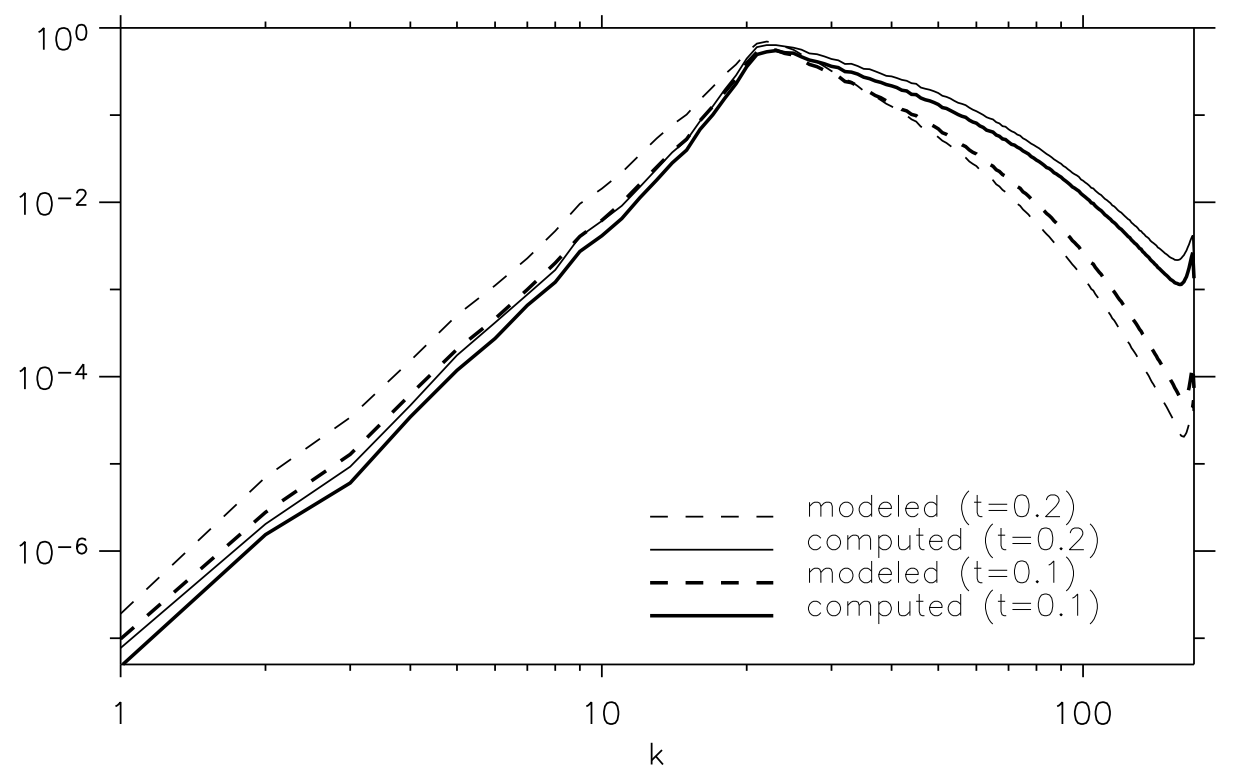

Figure 2: Comparison at two different times of the spectral density of $\mathbf{l}^{2}$ modeled by the integration of $\mathbf{l}$ (eq. 5) and the same quantity directly computed from the resolved and sub-grid scales $\left(\mathbf{l}=(\mathbf{U} \cdot \nabla) \mathbf{u}^{\prime}+\left(\mathbf{u}^{\prime} \cdot \nabla\right) \mathbf{U}\right)$.

the original RDT model. We therefore adopt this procedure as our starting RDT model, from which we now build our Langevin-LES model.

\subsection{Derivation of the model}

The derivation of the LES-Langevin model of turbulence proceeds in two steps. In the first one, we replace the term $\overline{\left(\mathbf{u}^{\prime} \cdot \nabla\right) \mathbf{u}^{\prime}}$ into a turbulent viscous term $\mu_{t} \Delta \mathbf{U}$, acting only at large scales, in the spirit of standard deterministic "eddy-viscosity" models. In a second step, we derive a suitable Langevin equation of the turbulent force $\mathbf{l}$ through a decimation of the number of degrees of freedom corresponding to scales beyond the aliasing limit. For this, we introduce a strong hyperviscosity to damp all components beyond a given cut-off wavenumber $k_{m}$. There is a priori complete freedom for the choice of $k_{m}$. Here, we note that the cascade-driven forcing $\mathbf{f}$ has components only up to $k=2 k_{c}$. Therefore, any component of 1 beyond $3 k_{c}$ will only be generated through secondary processes (stretching) rather than through the forcing. We may then hope that any $k_{m}$ between $2 k_{c}$ and $3 k_{c}$ provides the dominant contribution to the stochastic term 1 . We found that $k_{m}=2 k_{c}$ is in fact sufficient to capture this dominant contribution. Our Langevin-LES (LRDT) model is therefore finally given by:

$$
\begin{aligned}
& \partial_{t} \mathbf{U}+\overline{(\mathbf{U} \cdot \nabla) \mathbf{U}}+\overline{\mathbf{l}}=-\nabla P+\nabla\left(\nu+\mu_{t}\right) \nabla \mathbf{U} ;\left[0<k<k_{c}\right] \\
& \partial_{t} \mathbf{l}=-\left(\mathbf{l} / \tau_{f}\right)-(\mathbf{U} \cdot \nabla) \mathbf{l}^{\prime}-\left(\mathbf{l}^{\prime} \cdot \nabla\right) \mathbf{U} \\
& \quad+\nabla\left(\nu+\nu_{t}\right) \nabla \mathbf{l}+\xi_{\mathbf{0}}+\xi ;\left[0<k<2 k_{c}\right] \\
& \xi_{\mathbf{0}}=-(\mathbf{U} \cdot \nabla) \mathbf{f}-(\mathbf{f} \cdot \nabla) \mathbf{U}, \\
& <\xi>=\mathbf{0}, \quad<\xi_{i}(t, x) \xi_{j}\left(t^{\prime}, x^{\prime}\right)>=F_{i j}\left(t-t^{\prime}, x-x^{\prime}\right),
\end{aligned}
$$

where $\tau_{f}=27 / 22\left\langle\left(S_{i j} S_{i j}\right)^{1 / 2}\right\rangle, \nu_{t}$ and $\mu_{t}$ will be specified later and $f_{i}=\partial_{j}\left(U_{i} U_{j}-\overline{U_{i} U_{j}}\right)$. Looking at eq. (6), one recognizes a LES model where the backscatter coming from resolved scales-subfilter interaction is parameterized through a noise. The latter obeys a generalized Langevin equation, with friction made of viscosity and rapid distortion by resolved scales, and with stochastic forcing $\xi_{0}+\xi$, generated through the energy cascade and pressure processes. 
The RDT-based model we described is fully dynamics, and a priori adapted to any type of geometry, with possible anisotropies. In certain very simple cases, however, this model may support additional approximations, enabling a reduction in the computation cost. We present two examples below.

\subsubsection{Quasi-linear approximation (LQL)}

In the spirit of quasi-linear approximation, we may lump the transport and stretching by resolved scales with the viscous and friction term and simply replace them by a total friction term $-\mathbf{l} / \tau$, where $\tau$ is a typical time scale to be chosen later.

Note that this simplification somehow relies on the hypothesis that the advection and stretching of $\mathbf{l}$ by the resolved scales proceeds in an isotropic manner. We therefore do not expect this simple procedure to be fully valid in cases with e.g. stratification, or rotation, or walls. In the present case, it appears to give very good results, provided the time scale is chosen appropriately. This model therefore looks like:

$$
\begin{aligned}
& \partial_{t} \mathbf{U}+\overline{(\mathbf{U} \cdot \nabla) \mathbf{U}}+\overline{\mathbf{l}}=-\nabla P+\nabla\left(\nu+\mu_{t}\right) \nabla \mathbf{U} ;\left[0<k<k_{c}\right] \\
& \partial_{t} \mathbf{l}=-(\mathbf{l} / \tau)+\xi_{\mathbf{0}}+\xi ;\left[0<k<2 k_{c}\right]
\end{aligned}
$$

\subsubsection{The over-damped approximation (LQLOD)}

In situation where the time scale $\tau$ is very small, the damping in the Langevin equation is very large, and there is almost instantaneous adjustment of $\mathbf{l}$ to the forcing. This situation typically arises in locations where the resolved gradients are very large, i.e;. at the border of coherent resolved eddies. It is therefore interesting to run the model in this over-damped limit, to see whether these kinds of event dominate the overall dynamics. The resulting model (Langevin Quasi-Linear Over-Damped) looks like:

$$
\begin{aligned}
& \partial_{t} \mathbf{U}+\overline{(\mathbf{U} \cdot \nabla) \mathbf{U}}+\overline{\mathbf{l}}=-\nabla P+\nabla\left(\nu+\mu_{t}\right) \nabla \mathbf{U} ;\left[0<k<k_{c}\right] \\
& \mathbf{l}=\tau\left(\xi_{\mathbf{0}}+\xi\right) ;\left[0<k<2 k_{c}\right]
\end{aligned}
$$

Through this approximation, our model, based on the estimation of $\mathbf{l}$, becomes equivalent to the ADM velocity-estimation model of Domaradzki and collaborators [7, 5, 8. In a recent improvement of this model, the estimated small scales are used to integrate the Navier-Stokes equation on the fine grid over a given period of time short enough to prevent the pile up of the energy at the finest scales. The ADM model can therefore be viewed as a special case of our Langevin model. In the following, we show that this approximation perform very well and is indistinguishable from the general model in the isotropic homogeneous case. However, it may not be the case in more general situations, such as near walls. We leave this for future work.

\subsection{Parameters}

Our model includes three parameters $T_{i j}, \tau$ and $\mu_{t}$, that we now discuss. As previously discussed, the stochastic correlation $T_{i j}$ comes from pressure contribution, and we found its influence be irrelevant in the case with periodic boundary condition. In the present study, we therefore adopt $T_{i j}=0$, leaving its study for cases involving walls. For the time scale, we set $\tau=\tau_{f}+\alpha\left(S_{i j} S_{i j}\right)^{-1 / 2}$, so as to allow spatial variation of the friction time scale in agreement with local stretching. Alternatively, one may also use a global friction time by considering only spatial average of the local stretching rate, so that $\tau=\tau_{m}=\beta \tau_{f}$. This last prescription is actually better in term of computational cost. Empirically, we found that both prescriptions lead to a good model. In our numerical tests, we chose $\alpha=1$ or $\beta=1 / 2$ but we checked that the results are not very sensitive to these parameters

For the turbulent viscosity, we have an a priori rather vast choice, ranging from spectral to Smagorinsky models. In the present case, where our numerical scheme uses fast Fourier transform, the optimal choice (in term of computational cost) is the spectral model. The simplest choice is to link the turbulent viscosity to the kinetic energy at the cut-off wavenumber $k_{c}\left(\mu_{t} \propto \sqrt{E\left(k_{c}\right) / k_{c}}\right)$. The proportionality factor is tuned in our model and our numerical tests. Since part of the dissipation is produced by $\mathbf{l}$, the proportionality constant turns out to be reduced from 0.267 for the original spectral model (see 21]) to 0.08 in our case. Summarizing, we get the following parameters for our models: 


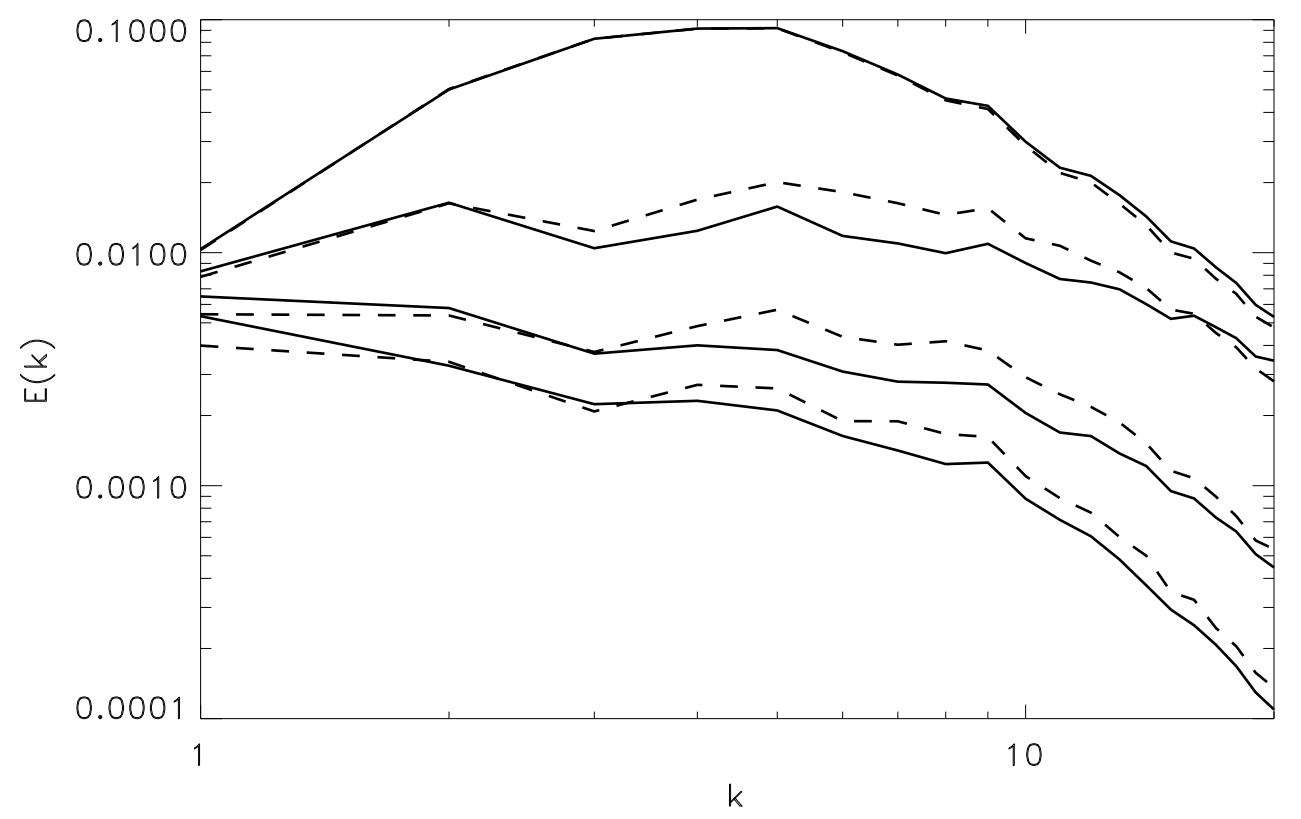

Figure 3: Energy spectra of the LRDT model (dash lines) and the equivalent DNS (continuous lines) of a decaying homogeneous isotropic turbulence. The spectra are compared for 4 different times: $t=(1,3,6,9)$.

$$
\begin{aligned}
& T_{i j}=0 \\
& \tau=(27 / 22)\left\langle\left(S_{i j} S_{i j}\right)^{-1 / 2}\right\rangle+\left(S_{i j} S_{i j}\right)^{-1 / 2} \\
& \tau_{m}=0.5\left\langle\left(S_{i j} S_{i j}\right)^{-1 / 2}\right\rangle \\
& \mu_{t}=0.08\left(E\left(k_{c}\right) / k_{c}\right)^{1 / 2}
\end{aligned}
$$

Models using the second prescription for the time scale will be called Langevin Quasi Linear Averaged (LQLA). They are optimal in term of computational cost.

\section{Model performances}

\subsection{Comparison of the Langevin models}

As a preliminary assessment of the interest of our model, we choose to test it in a simple configuration where we have both the code and the competence to implement it over a short time scale. This case corresponds to homogeneous, isotropic turbulence with periodic boundary conditions. It is clearly a restricted problem, where backscatter has proved to be secondary, and where eddy-viscosity based approaches perform very satisfactorily. In some sense, this ideal case is therefore a challenge to our model, for it is not clear whether there is room for further improvement with respect to eddy-viscosity description. In the following, we demonstrate a slight but discernible positive effect of our strategy.

The test is done both on decaying turbulence and forced turbulence in order to check the model ability to accurately reproduce the energy cascade from resolved to unresolved scales. The first comparison between the LRDT model (eq. 6) and the DNS is conducted over the energy spectra in fig. 3 and the

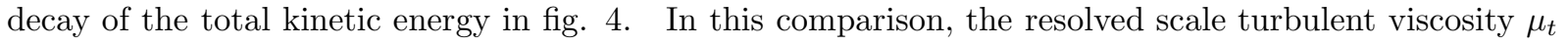
is tuned to give the best result in terms of energy spectra and decay of kinetic energy. The decimation of the small-scale of $\mathbf{l}$ (so as to keep only $2 k_{c}$ modes in each direction) is done through an hyper-viscosity $\left(\nu_{t}=0.002 k^{2} \sqrt{E\left(k_{c}\right) / k_{c}}\right)$ super-seeding the turbulent viscosity of the original RDT model. The energy spectra are in good agreement with the DNS for $t<1$ and slightly differ for the smallest resolved scales at later time. We are confident that a more elaborate tuning of the viscosity and friction term could have improved the model over later time, but such accuracy was not needed in the sequel. 


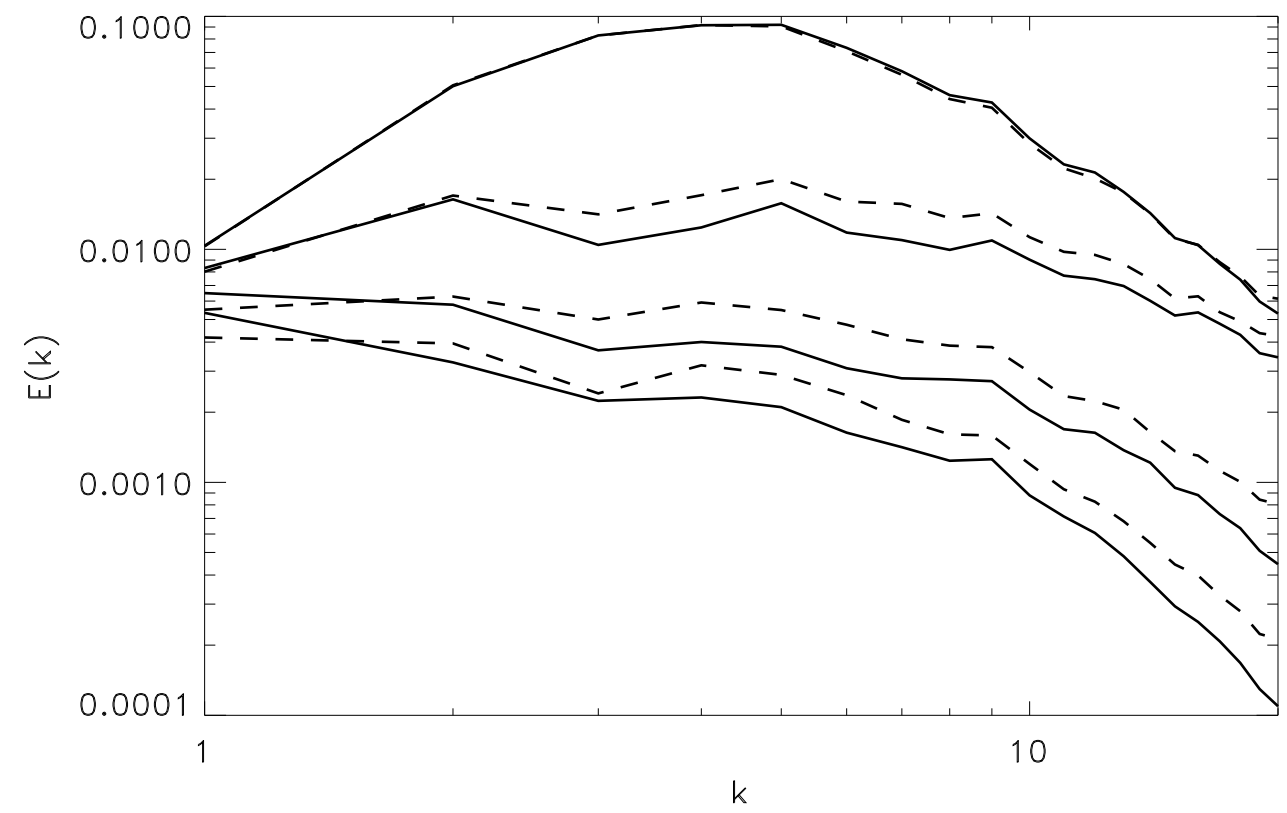

Figure 5: Energy spectra of the LQL model (dash lines) and the equivalent DNS (continuous lines) of a decaying homogeneous isotropic turbulence. The spectra are compared for 4 different times: $t=(1,3,6,9)$.

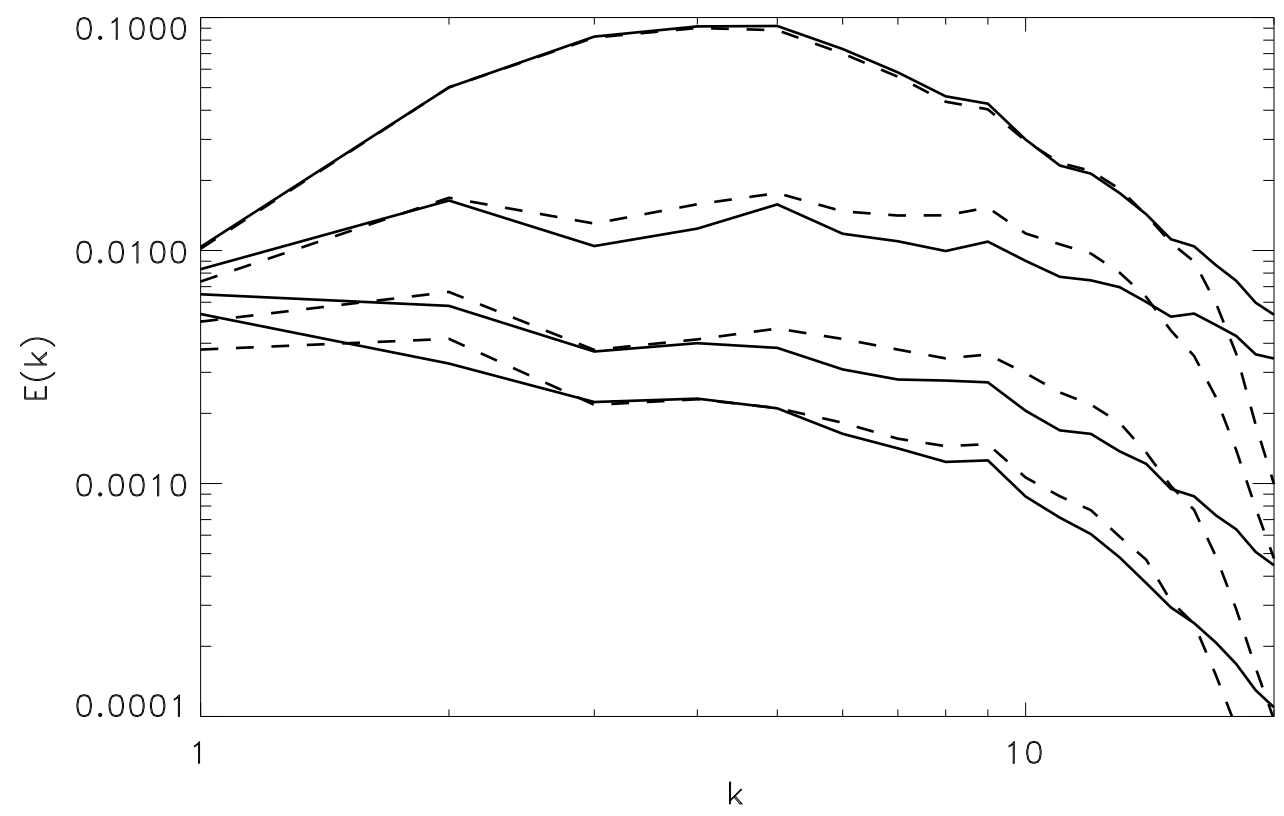

Figure 6: Energy spectra of the LQLOD model (dash lines) and the equivalent DNS (continuous lines) of a decaying homogeneous isotropic turbulence. The spectra are compared for 4 different times: $t=(1,3,6,9)$. 


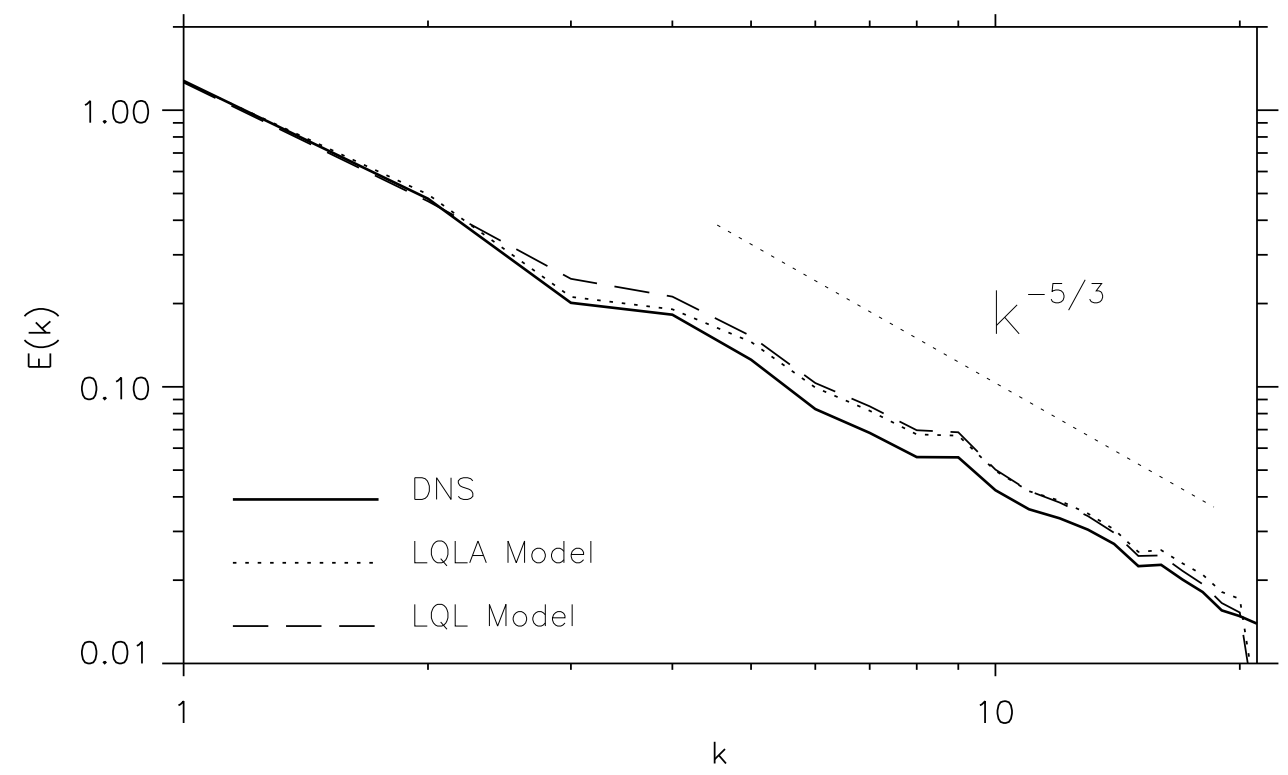

Figure 7: Averaged energy spectra for the simulation of forced isotropic homogeneous turbulence. The spectra are averaged over 4 turnover times for the DNS and 40 turnover times for the three LES. The DNS was performed with $384^{3}$ Fourier modes.

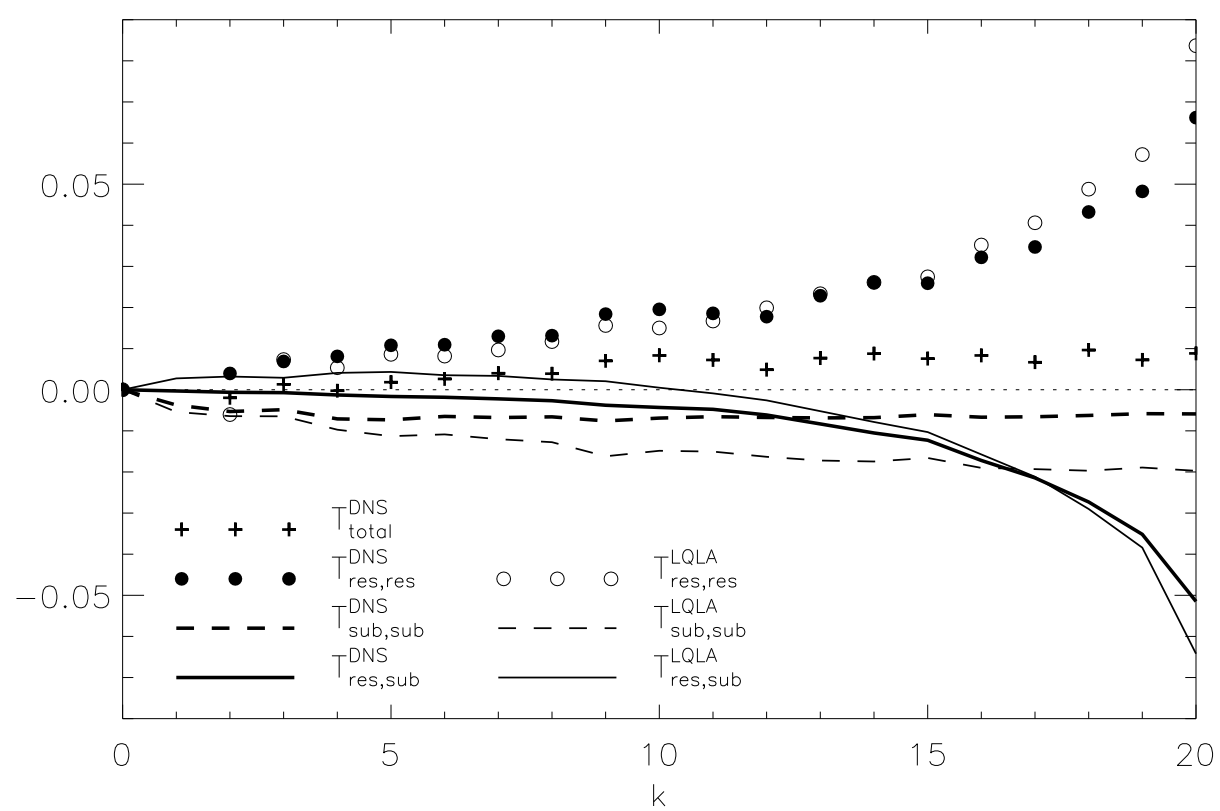

Figure 8: Comparison of the energy tranfer between the DNS and the LQLA model for forced isotropic turbulence. The energy tranfer is split into the 3 components involving resolved and subgrid scales. $T_{\text {res,sub }}^{L Q L A}$ is the energy transfer from the Lamb vector (see eq. 13) and $T_{s u b, s u b}^{L Q L A}$ is the disipation introduced by the turbulent viscosity $\mu_{t}$. 


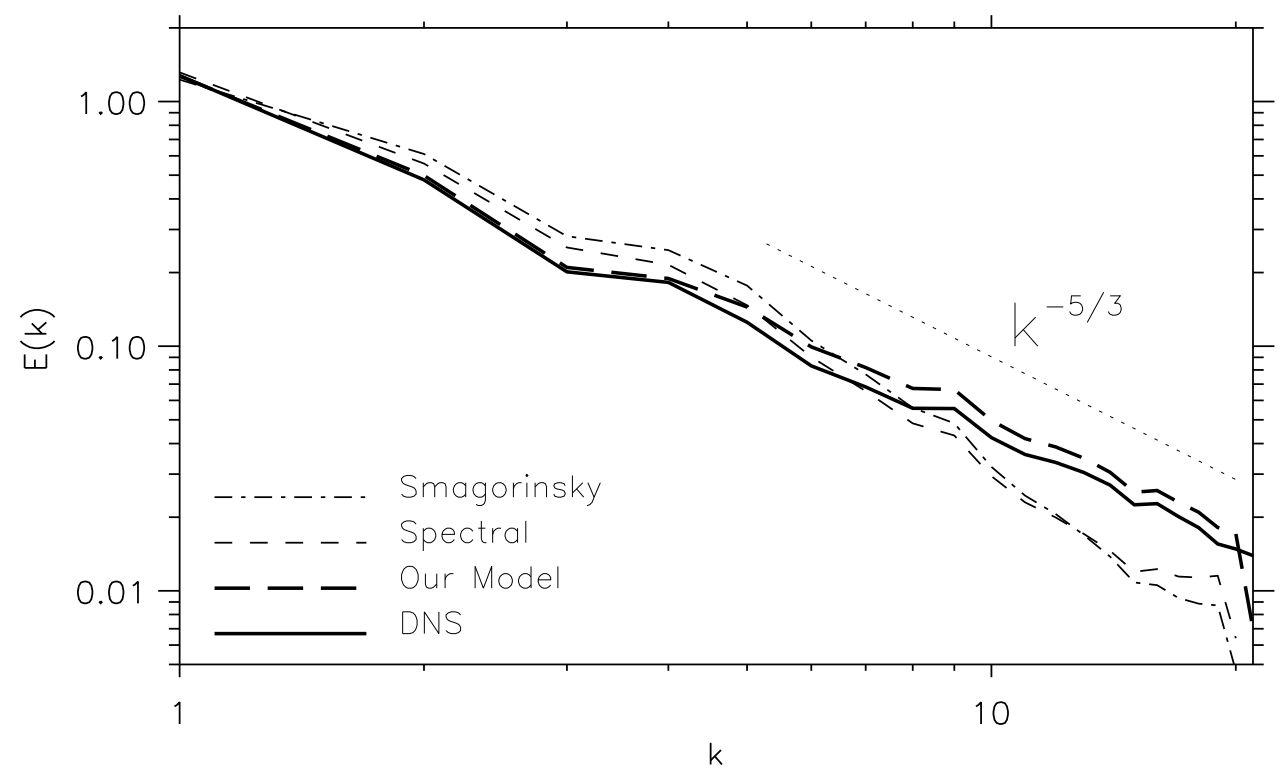

Figure 9: Comparison of the energy spectra of our LQLA model with the DNS, the Smagorinsky model and the spectral model (averaged over 4 turnover times for DNS and 40 turnover times for the 3 models)

\subsection{Comparison with other LES models}

Within the present framework (isotropic, homogeneous turbulence) it therefore seems that the LQLA model represents the best compromise in terms of accuracy and CPU cost. We now explore its performances with respect to other classical LES models in this field.

The simpler LES models for homogeneous, isotropic turbulence are based on eddy-viscosity concept, namely the Smagorinsky model [34] and the spectral model [21]. The later has only been tested for forced isotropic turbulence. The former corresponds to a Smagorinsky model (without dynamical procedure) in which the constant was tuned to get the best results in terms of energy decay. It was run on the finest grid of our model (the grid used for the integration of $\mathbf{l}$ ). The comparison is done using several statistical diagnostic, tracing different properties of the model.

\subsubsection{Energy spectra}

A comparison of the energy spectra for these two models and our LQLA model is shown in fig. [10 for the decaying case and in fig. 9 for the forced case. In the decaying case, our Langevin model performed at least as well as the Smagorinsky model in terms of energy decay. In the forced case, the Smagorinsky model clearly overestimate the energy dissipation at the smallest resolved scales, resulting in an energy spectrum much steeper than the theoretical $-5 / 3$ slopes. Similar result is found for the spectral model, while the LQLA model compares much better with the DNS at all scales. Indeed, our model seems to model properly the energy transfer at the largest scales and the energy dissipation near the cut-off.

\subsubsection{Skewness and flatness}

We also compared the skewness and the flatness of the LES models to the same statistics for the corresponding scales of the DNS in the case of forced turbulence. The results are displayed in table 1 The LQLA model gives a correct sign of the skewness, with a value three times too large, and a correct estimate of the flatness. By comparison, the Smagorinsky model predicts a wrong sign for the skewness and underestimates slightly the flatness. The spectral model gives the best estimate of the skewness (with correct sign) and underestimates the flatness of the distribution. 


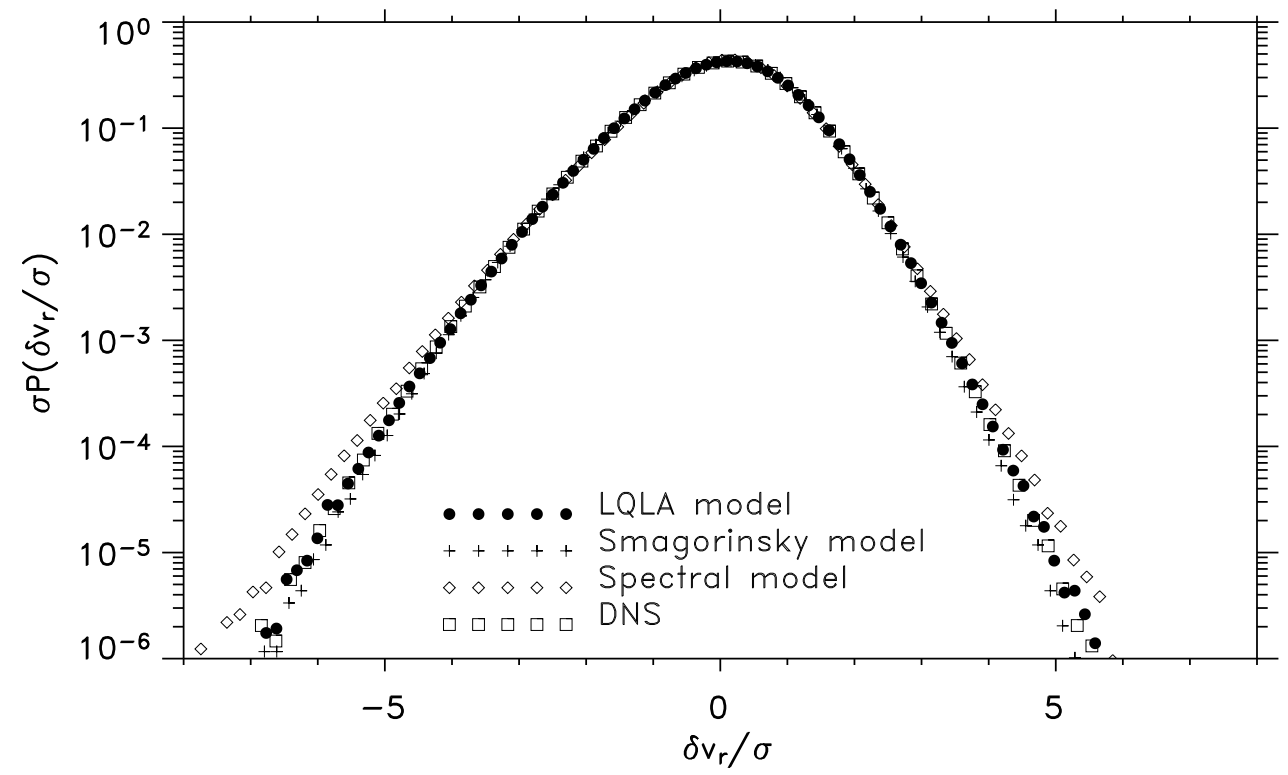

Figure 11: Comparison of the normalized PDF of longitudinal velocity increments $\left(\delta v_{r}=\mathbf{v}(\mathbf{x}+\mathbf{r})-\mathbf{v}(\mathbf{x})\right.$ with $|\mathbf{r}|=\frac{L}{64}$ and $\mathbf{r} \times \mathbf{v}=0$ ) for our LQLA model, the Smagorinsky model, the spectral model and the DNS. The PDF are averaged over 4 turnover times for DNS and 40 turnover times for the LES models.

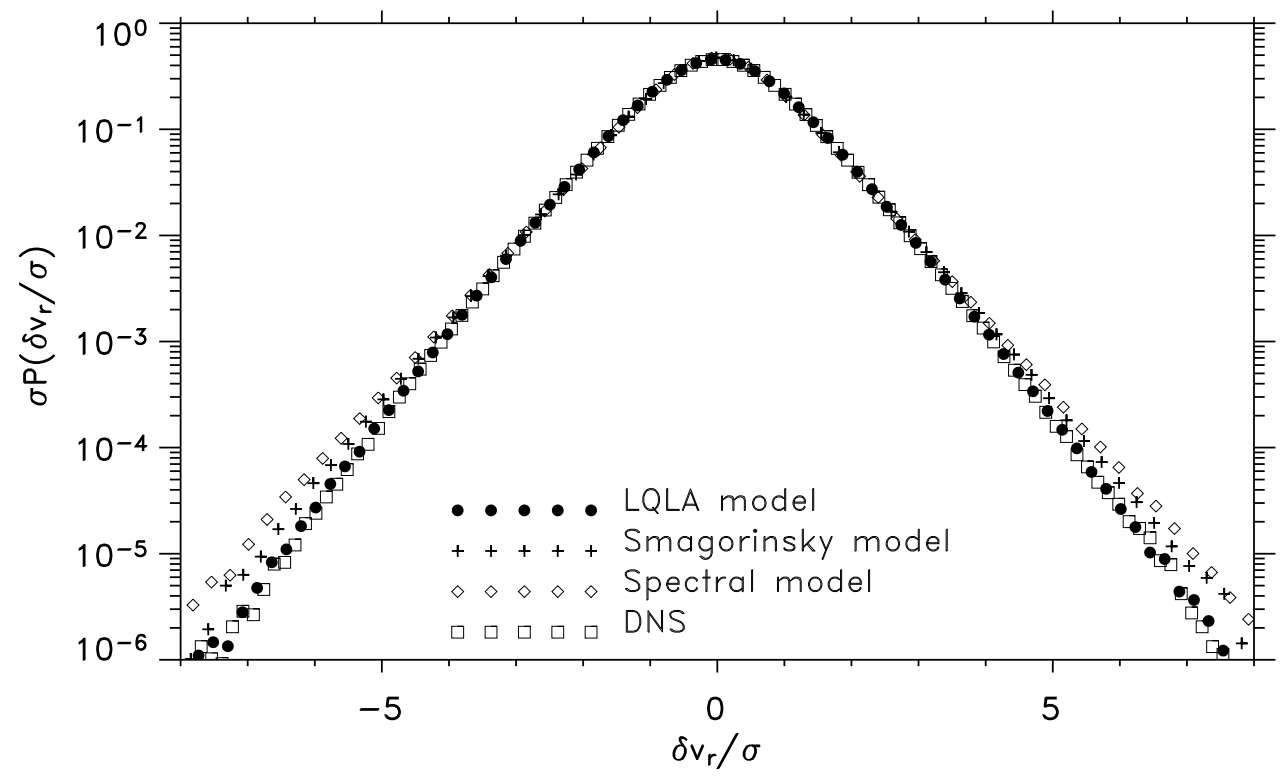

Figure 12: Comparison of the normalized PDF of transverse velocity increments $\left(\delta v_{r}=\mathbf{v}(\mathbf{x}+\mathbf{r})-\mathbf{v}(\mathbf{x})\right.$ with $|\mathbf{r}|=\frac{L}{64}$ and $\mathbf{r} \cdot \mathbf{v}=0$ ) for our LQLA model, the Smagorinsky model, the spectral model and the DNS. The PDF are averaged over 4 turnover times for DNS and 40 turnover times for the LES models. 


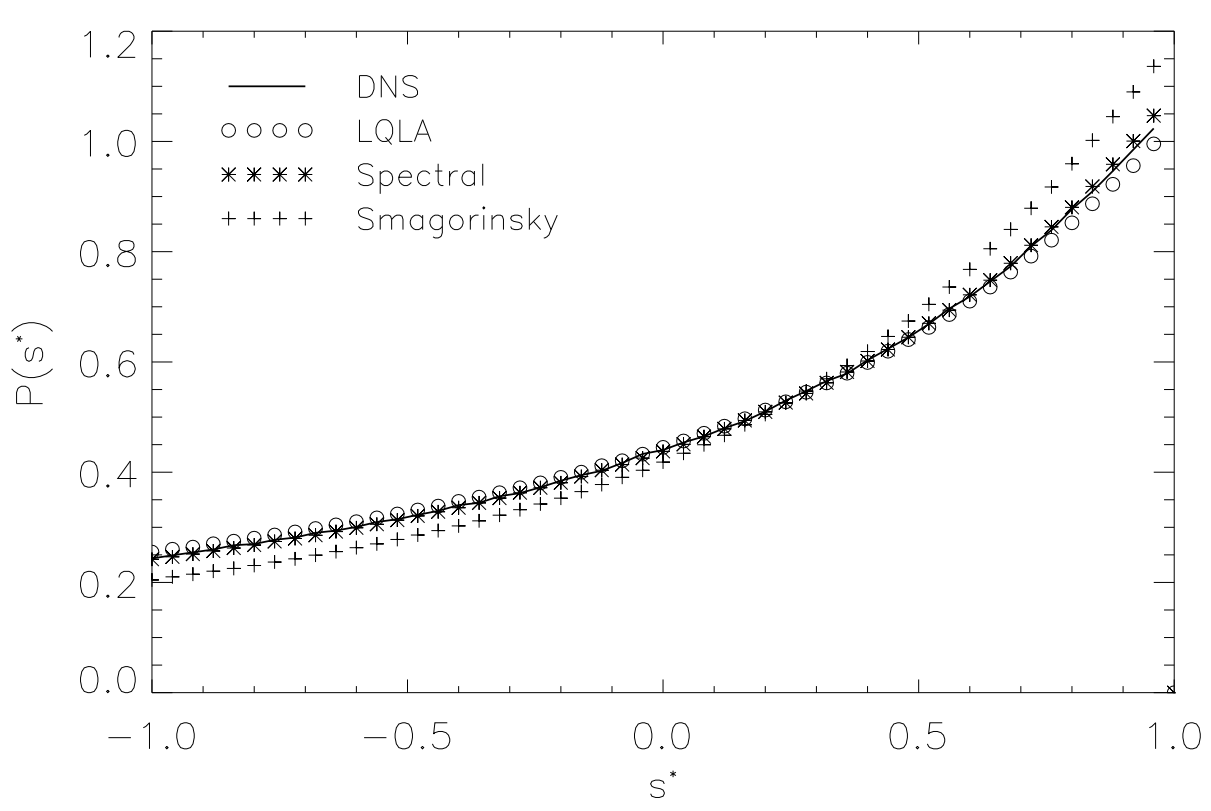

Figure 14: Comparison of the PDF of $s^{*}$ (eq. 18) for the resolved scales of the LQLA model, the Smagorinsky model and the spectral model and the corresponding DNS in the case of forced turbulence.

\subsubsection{Strain statistics}

Another important characteristic of the strain rate tensor $\mathrm{S}$ is the statistics of its eigenvalues. To quantify it, Lund and Rogers [24] proposed the following parameter:

$$
s^{*}=-3 \sqrt{6} \frac{\alpha \beta \gamma}{\left(\alpha^{2} \beta^{2} \gamma^{2}\right)^{3 / 2}}
$$

where $\alpha, \beta$ and $\gamma$ are the eigenvalues of $S$ such as $\alpha<\beta<\gamma$. The term $s^{*}$ measure the shape of the deformation caused by the strain rate tensor, with $s^{*}=-1,0,1$ corresponding respectively to worms, shear and pancakes. The probability density function of $s^{*}$ for the resolved scales of the LQLA model as well as for the Smagorinsky and the spectral models are computed in the case of forced turbulence. The comparison is shown on figure 14. The comparison shows very good agreement for both the LQLA model and the spectral model with respect to the DNS, while the Smagorinsky model shows poorer agreement.

\subsubsection{Computational cost}

The performance of each model can also be analyzed with respect to its computational cost. However, we are not able to give precise estimations of the performance because the implementation of each model was not optimized in terms of CPU cost. Moreover, the cost is highly related to the type of discretization and the numerical scheme. But, looking at the equations, we can expect an optimal computational cost for our model to be of the order of a DNS on a grid corresponding to the grid used for the integration of 1 . It as been shown that an integration with wavenumbers up to $2 k_{c}$ is enough for the equation of 1 . With the over-damped version of the model, there is no integration for $\mathbf{l}$ and we could expect a gain of a factor 2 relative to the time step.

\section{Discussion}

In this work, we present a new strategy to model subfilter scale in a Large-Eddy-Simulation. Our philosophy derives from the Brownian motion, where the extra degrees of freedom are modeled through the combination of a deterministic friction and stochastic forcing. Our model therefore includes both a deterministic eddy-viscosity and a stochastic turbulent force, solution of a generalized Langevin equation. 
motions, and stochastic energy backscatter from subfilter to resolved scale. Several model have been proposed in the past, to reproduce these two effects. At this point, it is interesting to review briefly the most popular ones, so as to understand their limitations and differences with respect to our model. A first example is the similarity model, which does not assume the alignment of the turbulent stress tensor with the strain rate tensor. In the original model of Bardina [1, the model of the Reynolds stress is achieved through approximation of the unfiltered velocity by the filtered velocity. This model can be seen as the zero-level of more general deconvolution models that rebuilds the unfiltered velocity (see [14 for a discussion on de-filtering in LES). This procedure however is also subject to serious limitations, since a LES with an exact deconvolution of the velocity field would lead to a simulation of the Navier-Stokes equation on a coarse grid without any model but with a sharp filter. Practically, the resulting lack of dissipation is provided through the physical or the numerical approximations of the deconvolution [35]. Other concepts arose from a detailed analysis of the energy transfer between resolved and subfilter scales. This leads to the conclusion that the scales smaller than half the smallest resolved scales have only few impact on the direct energy transfer to the largest scales 6, 40, 4,. Following this observation, a new method to capture the backscatter has been proposed through direct estimation or a more accurate integration of the largest subfilter-scales velocities. The simulations with these models can be accurate but expensive LES or cheap approximated DNS. The dynamics multilevel model [10, 36, 9] is an example of cheap DNS as it requires the same resolution as the corresponding DNS. Our 2D version of the RDT model [19] using a Lagrangian evolution of small-scale wave-packets is more flexible since the accuracy of the model is function of the number of modes used for the subfilter scales. Better performances in terms of computational cost can be obtained in models where only the largest unresolved scales are estimated. An example is provided by the ADM model of Domaradzki [7, 5, 8, where subfilter scales are estimated on a finer grid. The estimation of subfilter scales is performed after a first step of deconvolution. In a recent improvement of this model the estimated small scales are used to integrate the Navier Stokes equation on the fine grid over a given period of time short enough to prevent the pile up of the energy at the finest scales. Finally, one may note that attempt of direct inclusion of the backscatter have been done in the past through empirical addition of random numbers, with well chosen spectrum [20, 25]. These models led to noticeable improvement with respect to eddy-viscosity type of approach for boundary layer or plane shear mixing layer.

Most of these models have been validated and compared in several flow configurations. The most intensive comparison have been performed for isotropic turbulence where the DNS can reach higher Reynolds number. For instance, Fureby et al [13] made a comparative study of subgrid scale model of eddy-viscosity type, models of similarity type and Miles model. For moderate Reynolds number, they concluded that the difference between LES with different models are small but not insignificant. The characteristics of more specific type of models like eddy-viscosity models or similarity models have been studied intensively by many authors ([23, 27, 22]

From this review, it is clear that our originality is to seek for a systematic derivation of the stochastic effect, rooted in the Navier-Stokes dynamics. By this mean, we hope both to avoid uncontrolled, empirical modeling, and respect of all the symmetries of the original Navier-Stokes equation (a constraint not always easy to respect, see 29]). Our model may also easily be generalized to other more complicated systems, like e.g. rotating, stratified or inhomogeneous turbulence. Specifically, we separate the subfilter scale contribution into a term that correlates well to resolved strain tensors, and a term susceptible to the backscatter. The first term is modeled through a traditional eddy-viscosity, while the second is modeled through a noise, obeying a generalized Langevin equation. This equation is not postulated, but derived from the Navier-Stokes equation, through hypothesis akin to rapid distortion theory. The stochastic retroaction couples the resolved scales and the noise through the energy cascade, ensuring strong non-linearity of our model and non-trivial equilibrium solutions. A corner stone of our model is the estimation of $\mathbf{1}$, rather than the velocity, like in ADM model. Apart from this difference, there is some similarity between our model and the ADM model, in the simplest approximations we considered (quasi-linear and overdamped approximation). However, our model derives from the original Navier-Stokes equation through a well-defined and systematic procedure.

We have proposed a preliminary test of our model in isotropic, homogeneous situation, with periodic boundary conditions. In this case where the backscatter is secondary, we have shown that our model still perform slightly better than traditional LES approach used in this situation, based on eddy-viscosity concept. It would now be interesting to test our model in more realistic situations, with boundaries, where the stochastic modeling will become an essential ingredient. Given that our model reduces to the ADM model in a well-defined limit, we are confident that it can perform as well as this last model. The interesting question is whether our dynamical procedure increases its performance, and overcome certain limitations of the ADM model. This will be the subject of a future work. 
This work benefited from computer time allocated by IDRIS run by French CNRS and by CRIHAN (Centre de Ressource en Informatique de Haute Normandie, France) We thank the "Programme National de Planétologie", the GDR Turbulence and the GDR Dynamo for support. We have benefited from numerous discussion with our colleagues from GIT and from Lille University.

\section{References}

[1] J. Bardina, J. H. Ferziger, and W. C. Reynolds. Improved subgrid models for large eddy simulation. AIAA paper, 80:1357, 1980.

[2] R. Clark, J. Ferziger, and W. Reynolds. Evaluation of subgrid-scale models using an accurately simulated turbulent flow. J. Fluid Mech., 91:1-16, 1979.

[3] J. A. Domaradzki and N. A. Adams. Direct modelling of subgrid scales of turbulence in large eddy simulations. Journal of Turbulence, 3(024):1, 2002.

[4] J. A. Domaradzki, W. Liu, C. Härtel, and L. Kleiser. Energy transfer in numerically simulated wall-bounded turbulent flows. Phys. Fluids, 6:1583-1599, 1994.

[5] J. A. Domaradzki and K.-C. Loh. The subgrid-scale estimation model in physical space representation. Phys. Fluids, 11:2330-2342, 1999.

[6] J. A. Domaradzki, R. W. Metcalf, R. S. Rogallo, and J. J. Riley. Analisys of subgrid-scale eddy viscosity with use of results from direct numerical simulation. Phys. Rev. Lett., 58:547, 1987.

[7] J. A. Domaradzki and E. M. Saiki. A subgrid-scale model based on the estimation of unresolved scales of turbulence. Phys. Fluids A, 9:2148-2164, 1997.

[8] J. A. Domaradzki and P. P. Yee. The subgrid-scale estimation model for high Reynolds number turbulence. Phys. Fluids, 12(1):193-196, 2000.

[9] T. Dubois, F. Jauberteau, and R. Temam. Incremental unknowns, multilevel methods and the numerical simulation of turbulence. Computer method in applied Mechanics and Enineering, 159:123190, 1998.

[10] T. Dubois, F. Jauberteau, and R. Temam. Dynamical multilevel model for the simulation of turbulence. Cambridge University Press, Cambridge, UK, 1999.

[11] B. Dubrulle, U. Frisch, and M. Hénon. Low-viscosity lattice gases. J. Stat. Phys., 59:1187-1226, 1990.

[12] B. Dubrulle, J.-P. Laval, S. Nazarenko, and O. Zaboronski. A model for rapid stochastic distortions of small-scale turbulence. J. Fluids Mech., 520:1-21, 2004.

[13] C. Fureby, G. Tabor, H. G. Weller, and A. D. Gosman. A comparative study of subgrid scale models in homogeneous isotropic turbulence. Phys. Fluids, 9:1416-1429, 1997.

[14] M. Germano. Differential filters for the large eddy simulation of turbulent flows. Phys. Fluids, 29:1755-1778, 1986.

[15] K. Horiuti. A new dynamic two-parameter mixed model for large-eddy simulation. Phys Fluids, 9(11):3443-3464, 1997.

[16] E. T. Jaynes. Information theory and statistical mechanics. Physical Review, 106:620-630, 1957.

[17] J.-P. Laval, B. Dubrulle, and J. C. McWilliams. Langevin models of turbulence: Renormalization group, distant interaction algorithms or rapid distortion theory? Phys. Fluids, 15(5):1327-1339, 2003.

[18] J.-P. Laval, B. Dubrulle, and S. Nazarenko. Non-locality and intermittency in three-dimentional turbulence. Phys Fluids, 13(7):1995-2012, 2001.

[19] J.-P. Laval, B. Dubrulle, and S. Nazarenko. Fast numerical simulations of 2d turbulence using a dynamic model for subfilter motions. J. Comp. Phys., 196(1):184-207, 2004.

[20] C. E. Leith. Stochastic backscatter in a subgrid-scale mode: plane shear mixing layer. Phys. Fluids, 2:297-299, 1990.

[21] M. Lesieur. Turbulence in Fluids. 2nd edn. Kluwer, 1990.

[22] M. Lesieur and O. Métais. New trends in large-eddy simulations of turbulence. Ann. Rev. Fluid Mech., 28:45-82, 1996. 
from measurments in a turbulent jet. J. Fluid Mech., 275:83-119, 1994.

[24] T Lund and M. Rogers. An improved measure of the strain state probability in turbulent flows. Phys. Fluids., 6:1838-1847, 1994.

[25] P. J. Mason and D. J. Thomson. Stochastic backscatter in large-eddy simulations of boundary layers. J. Fluid Mech., 242:51-78, 1992.

[26] C. Meneveau. Statistics of turbulence subgrid scale stresses: necessary conditions and experimental tests. Phys. Fluids, 6:815-833, 1994.

[27] C. Meneveau and J. Katz. Dynamic testing of subgrid models in large eddy simulation based on the germano identity. Phys. Fluids, 11:245-247, 1999.

[28] C. Meneveau and J. Katz. Scale-inveriance and turbulence models for large eddy simulation. Annu. Rev. Fluid Mech., 32:1-32, 2000.

[29] M. Oberlack. Unified theory for symmetries in plane parallel turbulent shear flows. J. Fluids Mech., 427:299-328, 2001.

[30] S. Ossia and M. Lesieur. Energy backscatter in large-eddy simulations of the three dimensionnal incompressible isotropic turbulence. Journal of Turbulence, 1(010):1-11, 2000.

[31] U. Piomelli. Large-eddy simulation: achievements and challenges. Progress in Aerospace Science, $35: 335-362,1999$.

[32] S. B. Pope. Turbulent Flows. Cambridge Univ. Press, 2000.

[33] A Scotti and C. Meneveau. A fractal model for large eddy simulation of turbulent flow. Physica D, 127:198-232, 1999.

[34] J. Smagorinsky. General circulation experiment with the primitive equations, part i: The basic experiment. Mon. Wea. Rev., 91:99, 1963.

[35] S. Stolz and N. A. Adams. An approximate deconvolution procedure for the large-eddy simulation. Phys. Fluids, 11:1699-1701, 1999.

[36] R. Temam. Multilevel methods for the simulation of turbulence. a simple model. Journal of computational physics, 127:309-315, 1996.

[37] C. Tong, J. C. Wyngaard, S. Khanna, and J. G. Brasseur. Resolvable- and subgrid-scale measurement in the atmospheric surface layer: Technique and issues. Journal of Atmospheric Science, 55(20):31143126, 1998.

[38] F. van der Bos, C. Meneveau, and J. Katz. Effects of small scale turbulent motions on the filtered velocity gradient tensor as deduced from holographic particleimage velocimetry measurements. Phys. Fluid, 14(7):2456-2474, 2002.

[39] J.-Z. Wu, Y. Zhou, X.-Y. Lu, and M. Fan. Turbulent force as a diffusive field with vortical source. Phys. Fluids., 11(3):627-635, 1999.

[40] Y. Zhou. Degrees of locality of energy transfer in the inertial range. Phys. Fluids A, 5:1092-1094, 1993. 